\title{
Authentic Leadership and Its Relation to Structural Empowerment and Work Environment Among Nurses
}

\author{
Soha Mamdouh Elkholy*, Mervat Ebrahim Aly El Dahshan, Hind Hassan Abd El Mageed \\ Nursing Administration Department, Faculty of Nursing, Menoufia University, Menoufia, Egypt \\ Email address: \\ mamdouh.soha@yahoo.com (S. M. Elkholy) \\ ${ }^{*}$ Corresponding author \\ To cite this article: \\ Soha Mamdouh Elkholy, Mervat Ebrahim Aly El Dahshan, Hind Hassan Abd El Mageed. Authentic Leadership and Its Relation to Structural \\ Empowerment and Work Environment Among Nurses. American Journal of Nursing Science. Vol. 9, No. 3, 2020, pp. 136-144. \\ doi: 10.11648/j.ajns.20200903.21
}

Received: April 8, 2020; Accepted: May 3, 2020; Published: May 14, 2020

\begin{abstract}
Background: Nursing work environments often expose nurses to toxic behaviors. Today's nurse leaders face many challenges and concerns in order to empower their staff through creating professional practice environment that require a new type of leadership in which authentic leadership is considered a positive relational-leadership approach, a. Aim: To examine nurses' perspective of authentic leadership and its relation to structural empowerment and work environment in critical care units at Shebin El-Kom teaching hospital. Subjects and Methods: Non-experimental descriptive correlational research design was employed in the conduction of this study. A simple random sample (244) of nurses having minimum one year of experience composed the study sample. Three standardized questionnaires were utilized which are Authentic Leadership Inventory, Conditions of Work Effectiveness Questionnaire and Practice Environment Scale of the Nursing Work Index. Results: The current study declared that the majority $(98.4 \%)$ of nurses considered their leaders to be practicing moderate to high authentic leadership. Moreover, (95.1\%) of nurses experienced moderate to high levels of structural empowerment. Likewise, about three quarters (75.4\%) of nurses perceived high level of work environment. Conclusion and Recommendations: There was a significant positive relation among authentic leadership, structural empowerment and work environment from nurses' perspective. This study can help policy makers, administrators, and nursing leaders to detect areas in need of improvement in nurses' work environment and handle it, which leads to improvement in quality of nursing care and consequently patient outcomes.
\end{abstract}

Keywords: Authentic Leadership, Structural Empowerment, Work Environment

\section{Introduction}

Demands for nursing personnel continue to rise due to a predicted nursing shortage exceeding 1,000,000 nurses. Nurses leave institutions due to unsatisfactory work environment. Nurse executives have the opportunity to implement structure and processes to influence the work environment. The American Association of Critical Care Nurses has emphasized the integration of authentic leadership [1]. Authentic leaders are considered a self-aware individual applying their values and beliefs in the organization. Positive impact of authentic leaders on followers was acknowledged by most studies [2].

The theory of authentic leadership highlights the leader's sense of self, sharing values, and acting with consistency and transparency and also the influence on followers' attitudes and behaviors as cultivated through the leader's attitudes and behaviors. If the leader positively impacts the follower, then there is an indication of a willingness and ability to work. The work on the theory actually began in 1967 and had renewed interest and substantiation in the early 2000s as a desire for ethical leaders emerged in society along with a prevalence of mandated corporate ethics programs [3].

The authentic leadership includes four dimensions: (a) self-awareness, in which leaders understand their strengths and weaknesses and motivating others; (b) balance processing, signifies that leaders analyze all relevant data before taking a decision; (c) internalized moral perspective, states how leaders make decisions according to values and high internal ethical standards; and (d) relational 
transparency, which denotes how leaders are open in presenting one's true self to others [4].

Self-awareness: Refers to understanding of how one derives and makes meaning of the world and how it impacts the way one views himself over time. Also, showing an understanding strength, weaknesses, and the multifaceted nature of the self, which includes having insight, into the self through exposure to others, and being cognizant, of one's impact on other people [5].

Balanced processing: Authentic leaders practice balanced processing, which means that the leader objectively analyzes data or information before deciding and asks others for input, and solicits ideas from their followers to obtain diverse ideas and feedback as needed. Authentic leaders openly receive the views from others. By listening to others, an authentic leader attracts more followers, and by doing so, limits the damage caused by biases or exaggeration [6].

Internalized moral perspective: The term "internalizes moral perspective" refers to the observation that an authentic leader practices self-regulation guided by their own moral values, standards, and beliefs. Thus, they make decisions depending on own principles and perspectives and manage organizational pressures appropriately. Furthermore, such leaders are strong when handling external attempts at persuasion and when confronted with difficult decisions because of their own firmly-held values and standards [7].

Transparency: Authentic leaders practice transparency by disclosing their values and beliefs to their followers. Followers are critical to what they see and hear. Thus, transparency promotes emotional and psychological stability among followers that promotes the feeling of trust with one another. Moreover, leader who practices being transparent to his or her staff encourages trust, the making of good, solid, and consistent decisions [8].

Authentic leaders should provide an empowerment climate to gain positive outcomes, facilitate information access, support, resources, providing opportunities to learn and develop nurse [9]. Structural or workplace empowerment was first discussed by Kanter (1977), it is a framework in which empowerment promotes strong and effective communication, social interaction, and participation. Kanter's work is based on the premise that empowerment is derived from the working environment. Moreover, in order for nurses to be empowered, there are four components to be accessed: Information about all aspects of the organization; support in performing job and decision making; opportunity to participate in other areas of the organization and for promotion; and resources as needed must be present in the work environment [10].

Nonetheless, the favorable nursing practice environment construct is difficult to define and operationalize. It was described as a system which makes it possible to control the provision of nurse's healthcare and work environment. The term work environment is used to describe the surrounding conditions in which an employee works, it could be composed of physical conditions, such as office lighting or equipment, such as computers, it could be also linked to issues such as work processes or procedures; it can involve the social interactions at the workplace, including interactions with peers, subordinates, and managers [11].

Nursing practice environment excellence is vital to improve health care system performance. Work environment that facilitate full expressing nurses' skills and knowledge is needed [12]. Empowerment is an indicator of positive work behaviors and better work environment. There has been conducted program that investigated the impact of nursing work environments on nurses' empowerment for professional practice, their health and well-being, it reflected the leadership roles in creating empowering work conditions. Growing leadership qualities in followers through empowerment, encouragement, and support are hallmark characteristics of authentic leaders. Furthermore, authentic leadership enhances the sense of self-awareness and positive behaviors not only in the leaders, but in followers as well [13].

In addition, healthy nursing work environments are paramount to allow nurses to provide quality patient care. Nurses who work in a toxic work environment have emotional impacts caused by anxiety, mood swings, low selfesteem and depression. Moreover, empowered work environments are the antecedent to nurse perceptions of a supportive professional practice environment [14].

\subsection{Significance of the Research}

As leadership research in nursing had emerged and the desire to improve healthcare has intensified, the quest to explain and develop exemplary leadership behaviors has increased [15]. Leader behavior impacting the work environment and affecting nursing performance can either help or hinder nurses in fulfilling their purpose of quality patient care. The nurse executive has controlled the environment for patient care delivery. For nurse's satisfaction with work environment, the environment needs to be supported and empowered. In addition to allowing productive work, and preventing their departure from the organization [1].

Based on researchers' clinical experience through observing nursing departments. The nurses' performance and providing quality patient care affected by leadership style used in the hospital. Furthermore, findings of the reviewed literature identified limited studies done locally about authentic leadership. The field of authentic leadership is missing hard academic research to build what establishes effective authentic leadership and its relation to structural empowerment and work environment among nurses. Hence, it is important to fill the current research gap by conducting a study locally to examine nurses' perspective of authentic leadership and its relation to structural empowerment and work environment.

\subsection{Aim of the Research}

The aim of the current study was to examine nurses' perspective of authentic leadership and its relation to structural empowerment and work environment in intensive care units at Shebin El-Kom teaching hospital. 


\subsection{Research Questions}

1. What are authentic leadership levels from nurses' perspective?

2. What are structural empowerment levels from nurses' perspective?

3. What are work environment levels from nurses' perspective?

4. Is there a relationship among authentic leadership, structural empowerment and work environment from nurses' perspective?

\section{Subjects and Methods}

\subsection{Research Design}

Non-experimental descriptive correlational research design was employed in the conduction of this study.

\subsection{Setting}

The present research was implemented at Shebin El-Kom teaching hospital, Menoufia governorate, which is affiliated to General Organization for Teaching Hospitals and Institutes.

\subsection{Subjects}

A simple random sample (244) of nurses with minimum one year of experience composed the study sample. They are working in the following areas: (Intensive Care Units (ICU); Operating Room (OR); Obstetrics and Gynecology and Hemodialysis Unit).

\subsection{Data Collection Tools}

Three instruments were applied in this research in addition to the socio- demographic data. These were the Authentic Leadership Inventory (ALI); The Practice Environment Scale of the Nursing Work Index and Conditions of Work Effectiveness Questionnaire.

Tool I: It consisted of two sections as the following:

Section I: Socio- demographic data:

This instrument was designed by the researchers to gather the socio- demographic data of the participant nurses (such as; age, sex, marital status and years of experience).

Section II: The Authentic Leadership Inventory (ALI):

The Authentic Leadership Inventory (ALI) is designed to assess nurses' opinion about their leaders' characteristics. This tool was created by Neider \& Schriesheim, [16]. It is a self-administered questionnaire of 14 descriptive items. Each item is rated on a five-point Likert scale ranging from ' 1 ' (Strongly Disagree) to ' 5 ' (Strongly Agree). These items were divided into 4 subscales as follow; self-awareness (3 items); internalized moral perspective (4 items); balanced processing (4 items) and relational transparency (3 items).

The total score of $\mathrm{AL}$ was ranged from $(14-70)$, which is the sum of all nurses' responses in this scale. Scores from (14 - 41) points were considered as "low authentic leadership", scores from (42-55) were denoted as "moderate authentic leadership", and scores from (56-70) were considered as "high authentic leadership. The Cronbach's alpha coefficient was ranging from $(0.70-0.85)$.

Tool II: Conditions of Work Effectiveness Questionnaire

The Conditions of Work Effectiveness Questionnaire was used to assess nurses' structural empowerment. It was developed by Laschinger et el., [17]. I t consists of 19 questions which answered in a 5 Likert-type fashion with a scale ranging from $1-5,1$ and $2=$ None, 3 and $4=$ Some and $5=$ A Lot. The Empowerment total score is derived from summing of the six subscales: Opportunity (3 items); Information (3 items); Support (3 items); Resources (3 items); formal power (3 items) and finally informal power (4 items). The higher the score, the more structural empowerment the nurses experience.

The scores were then calculated and categorized as follows; scores $(<60 \%)$ were considered as "low structural empowerment", scores from $(60-75 \%)$ were considered as" moderate structural empowerment", and scores $(>75 \%)$ were considered as "high structural empowerment". Validity and Reliability of the CQEW-II has been verified carefully in nursing, and is constant in reliability and validity [18]. Reliability for this tool is established through Cronbach's alpha. Subscales scores range from $(0.78-0.93)$ [19]. Moreover, $(0.71-0.85)$ for subscales, and $(0.80)$ for total scale [20].

Tool III: Practice Environment Scale of the Nursing Work Index:

The Practice Environment Scale of the Nursing Work Index (PES-NWI) is used to assess the nurses' opinions about nursing work environment. The tool was created by [21]. It consists of 31 descriptive items. Each item is rated on a fivepoint Likert scale where $1=$ strongly disagree to $5=$ strongly agree. These items were divided into 5 subscales as follow: Nurse participation in hospital affairs (9 items); nursing foundations for quality of care (10 items); nurse manager ability, leadership, and support of nurses (5 items); staffing and resource adequacy (4 items); and finally collegial nursephysician relations (3 items).

The PES-NWI total score was calculated by adding sum of all the 31 items, and it ranged from $(31-124)$. Nurses who had a total score from $(31-77)$ points = "low level of nursing work environment, and those who had a total score from $(78$ - 124) points = "high level of nursing work environment. A higher score indicates better nursing work environment. The Cronbach's alpha coefficient for the total scale was 0.91 and the sub-scale coefficients ranged from 0.67 to 0.79 .

\subsection{Pilot Study}

A pilot study was performed on 20 nurses not included in research sample to examine the practicability and applicability of the study tools, identify any difficulties, and estimate the time required to fill in the questionnaire. Based on the results of the pilot study, the necessary alteration and clarification of some questions were done. 


\subsection{Methods of Data Collection}

An agreement to conduct the study was obtained from both medical and nursing executives of Shebin El-Kom teaching hospital, Menoufia governorate, Egypt. After that, an oral consent was taken from all nurses, then the researchers gave the questionnaire for filling it out, telling them that all information gathered will be used only for the purpose of research, and results of the study will be published in aggregates. Each participant spent 15-20 minutes to complete the questionnaire. The data collection stage of the study was applied in three months from $1 / 11 / 2019$ to $30 / 1 / 2020$.

\subsection{Ethics and Human Rights}

An oral consent was gained from all participant nurses before collecting any data. The data was collected by the researchers after describing the study aim to all participants. Anonymity and confidentiality of participants' information was totally guaranteed. Voluntary participation in the study was assured to all participants as well. They were informed about their rights to withdraw from the study at any time without giving any reason.

\subsection{Data Analysis Plan}

Data were revised, implied, entered, examined and tabulated using Statistical Package of Social Sciences (SPSS) version 23. Both descriptive statistics (frequency, percentage, mean and standard deviation) and inferential statistics (Pearson correlation test, chi-square test. $\mathrm{P}$ value less than 0.05 was considered significant.

\section{Results}

Table 1 showed the socio-demographic characteristics of participant nurses. The table indicated that the majority $(88.5 \%)$ of participant nurses were female, most of them (76.6\%) were married and near half $(44.4 \%)$ of them had Bachelor's degree in nursing and (44.2\%) of them had years of experience from (10 - 14 years).

Table 2. Demonstrated descriptive statistics of authentic leadership, structural empowerment and nursing work environment dimensions among nurses. Regarding authentic leadership, the total mean and SD was $(55.5 \pm 5.0)$. While, the total mean and SD of structural empowerment was (67.32 11.1). Concerning, nursing work environment, the total mean score and SD was $(87.47 \pm 18.5)$.

Figure 1. Showed the distribution of authentic leadership, structural empowerment and work environment from nurses' perspective. In relation to authentic leadership, it demonstrated that more than half $(54.1 \%)$ of participant nurses considered their leaders to be practicing high authentic leadership. While, in relation to structural empowerment, it revealed that around two third (59\%) of nurses had moderate level of structural empowerment. Moreover, nursing work environment from nurses' perspective, approximately, about three quarters $(75.4 \%)$ of nurses perceived high level of work environment.
Table 3. Showed significant positive relation among selfawareness of AL dimension and opportunity $(\mathrm{r}=.399)$, informal power $(\mathrm{r}=.347)$, and total structural empowerment $(\mathrm{r}=.375)$, while there was significantly negative correlation with both resources $(r=-285)$ and formal power $(r=-.270)$ structural empowerment dimensions. Regarding moral perspective dimension of $\mathrm{AL}$, it was significantly positive correlated with structural empowerment dimensions (support $(\mathrm{r}=.171)$, resources $(\mathrm{r}=.361)$, formal power $(\mathrm{r}=.306)$, informal power $(\mathrm{r}=.325)$, and total empowerment $(\mathrm{r}=.381)$. Also, relational transparency of AL dimension was significantly correlated with only formal power $(\mathrm{r}=.136)$ and informal power $(\mathrm{r}=.129)$ of structural empowerment dimensions $(\mathrm{P}<0.001)$. While there was no significant relation between balanced process of AL dimension and all structural empowerment dimensions.

Table 4. Showed significant positive correlation among moral behavior perspective dimension of $\mathrm{AL}$ and participation in hospital affairs $(\mathrm{r}=.319)$, foundation for quality of care $(\mathrm{r}=.352)$, manager ability, support \& leadership $(\mathrm{r}=168)$, and total work environment $(\mathrm{r}=.301)$. Regarding balanced process AL dimension, it was significantly positive correlated with manager ability, support $\&$ leadership $(\mathrm{r}=.312) . \quad(\mathrm{P}<0.001)$. While there was no significant correlation between self-awareness and relational transparency of $\mathrm{AL}$ dimension with all work environment dimensions.

Table 5. Revealed that there was a significant positive correlation among structural empowerment, work environment and authentic leadership among nurses. The correlation was stronger and highly significant between total score of empowerments, and authentic leadership among nurses $(\mathrm{P}<0.001)$, than between total score of work environment, and authentic leadership among nurses $(\mathrm{p}<0.05)$.

Table 1. Socio -demographic Characteristics of Participant Nurses $(N=244)$.

\begin{tabular}{|c|c|c|}
\hline Socio demographic characteristics: & N. & $\%$ \\
\hline \multicolumn{3}{|l|}{ Age (Years): } \\
\hline $20-29$ Years & 93 & 38.1 \\
\hline $30-39$ Years & 64 & 26.2 \\
\hline $40-52$ Years & 87 & 35.7 \\
\hline Mean \pm SD & \multicolumn{2}{|c|}{$33.7 \pm 9.5$ years } \\
\hline \multicolumn{3}{|l|}{ Educational Level: } \\
\hline Nursing School Diploma. & 106 & 43.4 \\
\hline Technical Institute of Nursing. & 60 & 24.6 \\
\hline Bachelor Degree in Nursing. & 78 & 32 \\
\hline \multicolumn{3}{|l|}{ Gender: } \\
\hline Male & 28 & 11.5 \\
\hline Female & 216 & 88.5 \\
\hline \multicolumn{3}{|l|}{ Marital status: } \\
\hline Married & 187 & 76.6 \\
\hline Unmarried & 57 & 23.4 \\
\hline \multicolumn{3}{|l|}{ Years of experiences: } \\
\hline $1-4$ years & 18 & 7.4 \\
\hline $5-9$ years & 82 & 33.6 \\
\hline $10-14$ years & 108 & 44.2 \\
\hline$\geq 15$ years & 36 & 14.8 \\
\hline Mean \pm SD & 12.5 & \\
\hline Units: & & \\
\hline
\end{tabular}




\begin{tabular}{lll}
\hline Socio demographic characteristics: & N. & \% \\
\hline Operating Room (OR). & 67 & 27.5 \\
Obstetrics \& Genecology (OR). & 43 & 17.6 \\
Intensive Care Units. & 83 & 34 \\
Haemodialysis Unit. & 51 & 20.9 \\
Total & 244 & 100 \\
\hline
\end{tabular}

Table 2. Authentic Leadership, Structural Empowerment and Work Environment Dimensions among Nurses $((N=244)$.

\begin{tabular}{lll}
\hline Items: & X & SD \\
\hline Authentic Leadership Dimensions: & & \\
Self-awareness & 12.18 & 1.5 \\
Internalized Moral Perspective. & 15.35 & 1.7 \\
Balanced Processing & 16.0 & 3.3 \\
Relational Transparency & 12.22 & 3.4 \\
Total Authentic Leadership: & 55.5 & 5.0 \\
Structural Empowerment Dimensions: & & \\
\hline
\end{tabular}

\begin{tabular}{lll}
\hline Items: & X & SD \\
\hline Opportunity. & 10.85 & 2.6 \\
Information. & 10.31 & 2.4 \\
Support. & 11.13 & 2.7 \\
Resources. & 9.33 & 2.8 \\
Formal Power. & 10.64 & 2.4 \\
Informal Power. & 15.06 & 2.3 \\
Total Structural Empowerment. & 67.32 & 11.1 \\
Nursing Work Environment Dimensions: & & \\
Nurse Participation in Hospital Affairs. & 25.11 & 6.7 \\
Nursing Foundations for Quality of Care. & 26.95 & 5.5 \\
Nurse Manager Ability, Leadership, and Support of & 14.68 & 5.6 \\
Nurses. & 12.13 & 4.2 \\
Staffing and Resource Adequacy. & 8.59 & 3.2 \\
Collegial Nurse-Physician Relations & 87.47 & 18.5 \\
Total Nursing Work Environment. & & \\
\hline
\end{tabular}

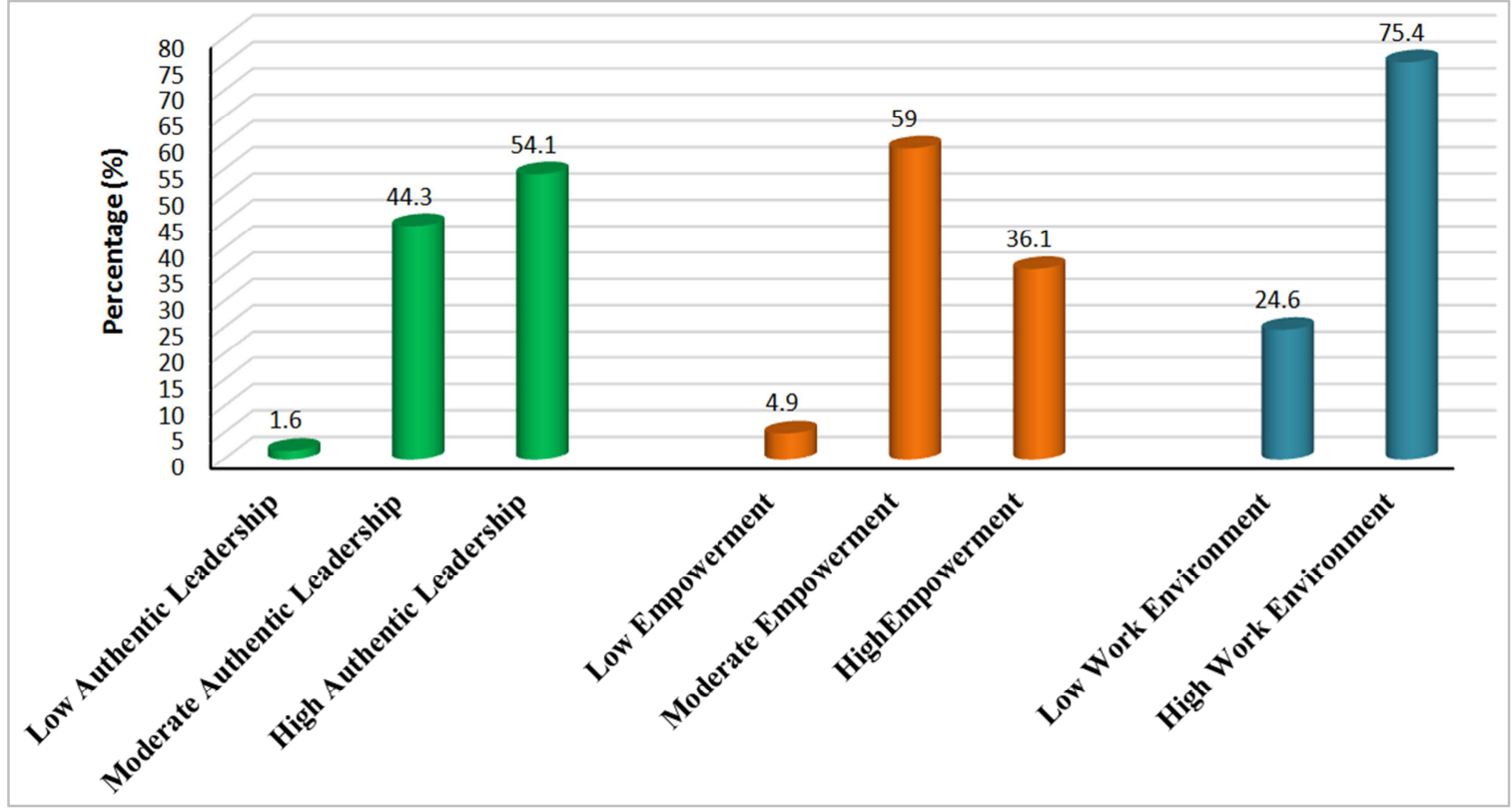

Figure 1. Distribution of Authentic Leadership, Structural Empowerment and Work Environment from Nurses' Perspective (N=244).

Table 3. Correlation Coefficient ( $r$ ) between Authentic Leadership Dimensions and Structural Empowerment Dimensions (N=244).

\begin{tabular}{|c|c|c|c|c|c|c|c|c|c|c|c|c|c|}
\hline Items: & & AL1 & AL2 & Al3 & AL4 & $\begin{array}{l}\text { Total } \\
\text { AL }\end{array}$ & Em1 & Em2 & Em3 & Em4 & Em5 & Em6 & $\begin{array}{l}\text { Total } \\
\text { Empowerment. }\end{array}$ \\
\hline Self-awareness (AL1) & $\begin{array}{l}\mathrm{r} \\
\mathrm{P}\end{array}$ & 1 & & & & & & & & & & & \\
\hline Molar Perspective (AL 2) & $\begin{array}{l}\mathrm{r} \\
\mathrm{P}\end{array}$ & $\begin{array}{l}.314 \\
.000\end{array}$ & 1 & & & & & & & & & & \\
\hline Balanced Process (Al 3) & $\begin{array}{l}\mathrm{r} \\
\mathrm{P}\end{array}$ & $\begin{array}{l}.151 \\
.018\end{array}$ & $\begin{array}{l}.261 \\
.000\end{array}$ & 1 & & & & & & & & & \\
\hline $\begin{array}{l}\text { Relational Transparency } \\
\text { (AL 4) }\end{array}$ & $\begin{array}{l}\mathrm{r} \\
\mathrm{P}\end{array}$ & $\begin{array}{l}-.106 \\
.098\end{array}$ & $\begin{array}{l}.125 \\
.052\end{array}$ & $\begin{array}{l}-.049 \\
.448\end{array}$ & 1 & & & & & & & & \\
\hline Total Authentic Leadership & $\begin{array}{l}\mathrm{r} \\
\mathrm{P}\end{array}$ & $\begin{array}{l}.494 \\
.000\end{array}$ & $\begin{array}{l}.700 \\
.000\end{array}$ & $\begin{array}{l}.609 \\
.000\end{array}$ & $\begin{array}{l}.475 \\
.000\end{array}$ & 1 & & & & & & & \\
\hline Opportunity (Em1) & $\begin{array}{l}\mathrm{r} \\
\mathrm{P}\end{array}$ & $\begin{array}{l}.399 \\
.000\end{array}$ & $\begin{array}{l}.121 \\
.060\end{array}$ & $\begin{array}{l}.106 \\
.100\end{array}$ & $\begin{array}{l}.004 \\
.955\end{array}$ & $\begin{array}{l}.250 \\
.000\end{array}$ & 1 & & & & & & \\
\hline Information (Em2) & $\begin{array}{l}\mathrm{r} \\
\mathrm{P}\end{array}$ & $\begin{array}{l}-.067 \\
.296\end{array}$ & $\begin{array}{l}.082 \\
.203\end{array}$ & $\begin{array}{l}-.075 \\
.243\end{array}$ & $\begin{array}{l}-.015 \\
.815\end{array}$ & $\begin{array}{l}-.032 \\
.616\end{array}$ & $\begin{array}{l}.507 \\
.000\end{array}$ & 1 & & & & & \\
\hline Support (Em3) & $\begin{array}{l}\mathrm{r} \\
\mathrm{P}\end{array}$ & $\begin{array}{l}.114 \\
.075\end{array}$ & $\begin{array}{l}.171 \\
.007\end{array}$ & $\begin{array}{l}-.108 \\
.092\end{array}$ & $\begin{array}{l}.049 \\
.448\end{array}$ & $\begin{array}{l}.106 \\
.099\end{array}$ & $\begin{array}{l}.655 \\
.000\end{array}$ & $\begin{array}{l}.401 \\
.000\end{array}$ & 1 & & & & \\
\hline Resources (Em4) & $\begin{array}{l}r \\
P\end{array}$ & $\begin{array}{l}-.285 \\
.000\end{array}$ & $\begin{array}{l}.361 \\
.000\end{array}$ & $\begin{array}{l}-.048 \\
.451\end{array}$ & $\begin{array}{l}-.039 \\
.548\end{array}$ & $\begin{array}{l}-.020 \\
.760\end{array}$ & $\begin{array}{l}.125 \\
.052\end{array}$ & $\begin{array}{l}.533 \\
.000\end{array}$ & $\begin{array}{l}.258 \\
.000\end{array}$ & 1 & & & \\
\hline Formal Power (Em5) & $\begin{array}{l}\mathrm{r} \\
\mathrm{P}\end{array}$ & $\begin{array}{l}.270 \\
.000\end{array}$ & $\begin{array}{l}.306 \\
.000\end{array}$ & $\begin{array}{l}-.002 \\
.979\end{array}$ & $\begin{array}{l}.136 \\
.034\end{array}$ & $\begin{array}{l}.299 \\
.000\end{array}$ & $\begin{array}{l}.413 \\
.000\end{array}$ & $\begin{array}{l}.115 \\
.073\end{array}$ & $\begin{array}{l}.535 \\
.000\end{array}$ & $\begin{array}{l}.260 \\
.000\end{array}$ & 1 & & \\
\hline
\end{tabular}




\begin{tabular}{|c|c|c|c|c|c|c|c|c|c|c|c|c|c|}
\hline Items: & & AL1 & AL2 & Al3 & AL4 & $\begin{array}{l}\text { Total } \\
\text { AL }\end{array}$ & Em1 & Em2 & Em3 & Em4 & Em5 & Em6 & $\begin{array}{l}\text { Total } \\
\text { Empowerment. }\end{array}$ \\
\hline \multirow{2}{*}{ Informal Power (Em6) } & $r$ & .347 & .325 & -.128 & .129 & .267 & .529 & .362 & .621 & .307 & .578 & 1 & \\
\hline & $\mathrm{P}$ & .000 & .000 & .046 & .044 & .000 & .000 & .000 & .000 & .000 & .000 & & \\
\hline \multirow{2}{*}{ Total Empowerment. } & $r$ & .375 & .381 & -.022 & .109 & .326 & .520 & .339 & .684 & .272 & .618 & .776 & 1 \\
\hline & $\mathrm{P}$ & .000 & .000 & .730 & .090 & .000 & .000 & .000 & .000 & .000 & .000 & .000 & \\
\hline
\end{tabular}

$\mathrm{Al}=$ Authentic Leadership, Em=Empowerment.

Table 4. Correlation Coefficient (r) between Authentic Leadership Dimensions and Nursing Work Environment Dimensions (N=244).

\begin{tabular}{|c|c|c|c|c|c|c|c|c|c|c|c|c|}
\hline Items: & & AL1 & AL2 & Al3 & AL4 & $\begin{array}{l}\text { Total } \\
\text { AL }\end{array}$ & En1 & En2 & En3 & En4 & En5 & $\begin{array}{l}\text { Total } \\
\text { Environ. }\end{array}$ \\
\hline Self-awareness (AL1) & $\begin{array}{l}\mathrm{r} \\
\mathrm{P}\end{array}$ & 1 & & & & & & & & & & \\
\hline $\begin{array}{l}\text { Molar behaviour (AL } \\
\text { 2) }\end{array}$ & $\begin{array}{l}\mathrm{r} \\
\mathrm{P}\end{array}$ & $\begin{array}{l}.314 \\
.000\end{array}$ & 1 & & & & & & & & & \\
\hline Balanced process (Al & $\begin{array}{l}\mathrm{r} \\
\mathrm{P}\end{array}$ & $\begin{array}{l}.151 \\
.018\end{array}$ & $\begin{array}{l}.261 \\
.000\end{array}$ & 1 & & & & & & & & \\
\hline $\begin{array}{l}\text { Relation transparency } \\
\text { (AL 4) }\end{array}$ & $\begin{array}{l}\mathrm{r} \\
\mathrm{P}\end{array}$ & $\begin{array}{l}-.106 \\
.098\end{array}$ & $\begin{array}{l}.125 \\
.052\end{array}$ & $\begin{array}{l}-.049 \\
.448\end{array}$ & 1 & & & & & & & \\
\hline Total authentic leadership & $\begin{array}{l}\mathrm{r} \\
\mathrm{P}\end{array}$ & $\begin{array}{l}.494 \\
.000\end{array}$ & $\begin{array}{l}.700 \\
.000\end{array}$ & $\begin{array}{l}.609 \\
.000\end{array}$ & $\begin{array}{l}.475 \\
.000\end{array}$ & 1 & & & & & & \\
\hline $\begin{array}{l}\text { Participation in } \\
\text { hospital affairs (En1) }\end{array}$ & $\begin{array}{l}\mathrm{r} \\
\mathrm{P}\end{array}$ & $\begin{array}{l}.022 \\
.729\end{array}$ & $\begin{array}{l}.319 \\
.000\end{array}$ & $\begin{array}{l}.013 \\
.836\end{array}$ & $\begin{array}{l}.028 \\
.660\end{array}$ & $\begin{array}{l}.152 \\
.018\end{array}$ & 1 & & & & & \\
\hline $\begin{array}{l}\text { Foundation for quality } \\
\text { of care (En } 2)\end{array}$ & $\begin{array}{l}\mathrm{r} \\
\mathrm{P}\end{array}$ & $\begin{array}{l}.120 \\
.060\end{array}$ & $\begin{array}{l}.352 \\
.000\end{array}$ & $\begin{array}{l}-.071 \\
.269\end{array}$ & $\begin{array}{l}.120 \\
.061\end{array}$ & $\begin{array}{l}.207 \\
.001\end{array}$ & $\begin{array}{l}.672 \\
.000\end{array}$ & 1 & & & & \\
\hline $\begin{array}{l}\text { Manager ability, } \\
\text { support \& leadership } \\
\text { (En3) }\end{array}$ & $\mathrm{P}$ & $\begin{array}{l}-.096 \\
.136\end{array}$ & .009 & .000 & $\begin{array}{l}-.052 \\
.422\end{array}$ & .024 & .000 & .355 & 1 & & & \\
\hline $\begin{array}{l}\text { Staffing and resource } \\
\text { adequacy (En4) }\end{array}$ & $\begin{array}{l}\mathrm{r} \\
\mathrm{P}\end{array}$ & $\begin{array}{l}-.026 \\
.681\end{array}$ & $\begin{array}{l}.003 \\
.961\end{array}$ & $\begin{array}{l}-.156 \\
.014\end{array}$ & $\begin{array}{l}-.053 \\
.409\end{array}$ & $\begin{array}{l}-.124 \\
.054\end{array}$ & $\begin{array}{l}.099 \\
.123\end{array}$ & $\begin{array}{l}.106 \\
.097\end{array}$ & $\begin{array}{l}.049 \\
.450\end{array}$ & 1 & & \\
\hline $\begin{array}{l}\text { Collegial nurse- } \\
\text { physician relations } \\
\text { (En5) }\end{array}$ & $\mathrm{P}$ & .938 & .015 & -.059 & -.001 & .634 & .681 & .000 & .606 & .031 & 1 & \\
\hline Total Environment & $\begin{array}{l}\mathrm{r} \\
\mathrm{P}\end{array}$ & $\begin{array}{l}.010 \\
.878\end{array}$ & $\begin{array}{l}.301 \\
.000\end{array}$ & $\begin{array}{l}.032 \\
.620\end{array}$ & $\begin{array}{c}.018 \\
.779\end{array}$ & $\begin{array}{l}.138 \\
.031\end{array}$ & $\begin{array}{l}.909 \\
.000\end{array}$ & $\begin{array}{l}.761 \\
.000\end{array}$ & $\begin{array}{l}.770 \\
.000\end{array}$ & $\begin{array}{l}.320 \\
.000\end{array}$ & $\begin{array}{l}.759 \\
.000\end{array}$ & 1 \\
\hline
\end{tabular}

$\mathrm{Al}=$ Authentic Leadership, En=Work Environment.

Table 5. Correlation Coefficient (r) among Authentic Leadership, Work Environment and Structural Empowerment from Nurses' Perspective (N=244).

\begin{tabular}{llll}
\hline & \multicolumn{2}{l}{ Nursing Work Environment } & Structural Empowerment \\
\cline { 2 - 4 } & $\mathbf{r}$ & $\mathbf{P}$ & $\mathbf{r}$ \\
\hline Authentic Leadership & 0.14 & $<0.05 \mathrm{Sig}$. & 0.22 \\
\hline
\end{tabular}

$\mathrm{LR}=$ Likelihood Ratio HS $=$ High significant $\mathrm{NS}=$ Not significant

\section{Discussion}

Authentic leaders are aware of their thinking and behavior and respect others' moral perspectives, knowledge, and strengths [22]. The American Association of Critical-Care Nurses [23] noted that the key element in creating a good work environment in nursing practice was authentic leadership. Likewise, nurse managers' leadership styles influenced nurses' thoughts, attitudes, and perceptions of their work. Moreover, authentic leadership can retain employees by creating trust and authentic relationships among members of the work group [24].

The result of this study declared that the majority (98.4\%) of nurses considered their leaders to be practicing moderate to high authentic leadership. This result was supported by Lee et al., [22] who revealed that the nurses considered their head nurses to be frequently applying authentic leadership. Authentic leadership had a direct positive effect on work environment. In the same track, the indices of the authentic leadership inventory were high with a cumulative score of $(87$,$) indicating a positive correlation of authentic leadership$ with employee engagement [25]. Also, Haddad [26] indicated moderate levels of authentic leadership but what remains unclear is what affects those scores and how the leader impacts feelings of empowerment, and professional practice environments.

The results of this study discovered that the majority (95.1\%) of nurses experienced moderate to high levels of structural empowerment. From researchers' point of view, this result can be clarified by the fact that nurses in intensive care units have an opportunity to develop their skill which make them find job opportunities in good places and travelling opportunities. In addition, the availability of resources; information; support and participation in decisions make them feel autonomous and empowered. This result was in the same tract with Regan et al., [27] who declared that 
nurses reported moderate perceptions of structural empowerment.

The finding of this study reported a significant positive correlation between authentic leadership, and structural empowerment among nurses. This result was in alignment with $X u$ and Yang [9] who mentioned that authentic leaders can enhance individuals' experience of empowerment by implementing empowering practices and creating an autonomous work environment, suggesting that empowerment originates from leadership and that increased empowerment can be achieved through enhancing authentic leadership.

Spence-Laschinger et al., [28] highlighting the vital role of authentic leadership in creating empowering work environments regardless of how experienced the followers are. This finding adds to literature knowledge linking nursing leadership to structural empowerment. Previous studies have found significant relationships between nurses' perceptions of structurally empowering conditions and a variety of styles of leadership, including emotionally intelligent leadership behaviors [29, 30]. Meanwhile, Laschinger et al., [31] showed that authentic leadership has a positive effect on group empowerment climate and team positive affective tone.

Moreover, managers effectively increase nurses' perceptions of workplace empowerment by focusing transparency, balanced processing, self-awareness, and high ethical standards which in turn enhances nurses' performance and job satisfaction. Work environments that provide open access to information, resources, support, and opportunities for learning and development both empower and enable nurses to accomplish their work [32].

The result of this study reported that there was a statistically significant correlation between of structural empowerment and authentic leadership all dimensions except balanced process. This result was in the same line with Spence-Laschinger et al., [28] who reported that total structural empowerment was significantly related to all aspects of authentic leadership in both groups, but most strongly to balanced processing and self-awareness, suggesting that these characteristics are particularly important to creating empowering work structures for followers. When leaders are perceived to be showing interest in employees' input, both positive and negative, for making important decisions that affect them, nurses are more likely to feel that their work environment is empowering and facilitates accomplishment of their work. These results suggest that regardless of how leadership is intellectualized, it is strongly projecting of empowerment. Furthermore, similar preceding results relating leadership behavior to structural empowerment [18, 33].

The results of this study discovered that nurses experienced high levels of nursing work environment as evident by means and SD $(87.47 \pm 18.5)$. Likewise, about three quarters of nurses perceived high level of work environment, while only one quarter of them had low level of nursing work environment. From researchers' point of view, this result may be explained by the fact that this study was carried out in the intensive care units, which have most of the hospital resources. Also, the majority of nurses hold a bachelor's degree, which makes them able to solve problems in a scientific approach and have a good relationship with physicians because of the convergence of their level of education. Furthermore, the critical condition of the patients raises the sympathy of the nurses and make them praise their God, do their best, and do not look at trivial things. In addition, when nurses experience a healthy relationship with coworkers, a supportive working environment, involvement in decision-making, and an increased level of success in clinical services, they perceive their work environment positively.

This finding was in the same line with Cheng et al., [34] who mentioned that the mean and SD of nursing practice environment score was $(91.33 \pm 9.04)$. Moreover, the finding was congruent with Al Moosa, et al., [12] who stated that the total mean score of practice environment was moderate $(\mathrm{M}=2.9, \quad \mathrm{SD}=0.47)$, considering the majority of the respondents are staff nurses who routinely work at the bedside with patients, this relatively high mean score suggests favorability of practice environments.

Furthermore, this result was consistent with Almuhsen et al., [11] who reported moderately high nurses' perception of work environment characteristics. In the same track, Lin et al., [35] revealed that participants earned an average work environment satisfaction score of 3.59, indicating that there remains significant room for improvement in practice environments and work conditions in hospitals in Taiwan.

Moreover, the study was support by Ozer et al., [36] who mentioned that the average number of points awarded by nurses using the work environment scale falls within the medium band. Likewise, a study directed by Ulusoy and Polatkan [37] revealed that the average number of points given by nurses on a work environment assessment scale was $(2.16 \pm 0.49)$. In other studies, nurses have evaluated their work environment positively $[38,39]$. On the contrary with the study's finding AL-Hadrawi et al., [40] revealed that the majority $(80 \%)$ of the surveyed nurses regarded their practice environment as poor. This reflects serious defects in both the structures and practice dynamic in the work environment.

The result of this study showed a significant positive correlation between authentic leadership, and work environment among nurses. Furthermore, the result was congruent with Lee et al., [22] who found a moderate and positive correlation between authentic leadership and positive work environment $(r=0.54)$. Authentic leaders can touch the hearts of employees, inspire their team members, create a healthy work environment. Moreover, awareness of the key characteristics of authentic leadership and the ability to master these can help the healthcare leader to build and grow an organizational culture that supports the healthcare worker in being engaged in this important work. When leaders are able to create a supportive and sustainable positive organizational climate, everyone wins [41].

The findings of this study shown that there was a positive 
significant correlation among authentic leadership, structural empowerment and work environment from nurses' perspective. This result was in the same line with SpenceLaschinger et al., [28] who mentioned that total structural empowerment was significantly related to all aspects of authentic leadership in both groups. Likewise, moderate correlation was found among empowerment, professional practice and authentic leadership [26]. Moreover, Regan et al., [27] contended higher levels of structural empowerment, authentic leadership, and the presence of a professional nursing practice environment. In addition, Khan et al., [42] noted that structural empowerment was vital to the work environment and organizational effectiveness.

\section{Conclusion and Recommendations}

This study participates in the current literature by investigating authentic leadership and its relation to structural empowerment and nursing work environment. The present study revealed that the majority $(98.4 \%)$ of nurses considered their leaders to be practicing moderate to high authentic leadership. Moreover, (95.1\%) of nurses experienced moderate to high levels of structural empowerment. Likewise, about three quarters $(75.4 \%)$ of nurses perceived high level of work environment. Also, there was a significant positive relation among authentic leadership, structural empowerment and work environment from nurses' perspective.

This study can help policy makers, administrators, and nursing leaders to detect areas in need of improvement in nurses' work environment and handle it, which leads to improvement in quality of nursing care and consequently patient outcomes. Further researches are required on authentic leadership with a larger sample size extending across diverse sectors in different governorate to generalize the findings.

\section{Acknowledgements}

It is a great pleasure to thank all nurses for their cooperation and help during data collection.

\section{References}

[1] Goerke, K. B. (2019). Correlation of Nurse Executive Authentic Leadership Attributes with Work Environment in Acute Care Hospitals (Doctoral dissertation, Grand Canyon University).

[2] Anwar, A., Abid, G. and Waqas, A. (2020). Authentic Leadership and Creativity: Moderated Meditation Model of Resilience and Hope in the Health Sector. European Journal of Investigation in Health Psychology and Education; 10: 18-29.

[3] Frisch, C. \& Huppenbauer, M. (2014). New insights into ethical leadership: A qualitative investigation of the experiences of executive ethical leaders. Journal of Business Ethics, 123 (1), 23-43. doi: 10.1007/s10551-013-1797-9.
[4] Salcedo, J. F. (2016). Authentic Leadership: A Quantitative Study of the Effect of Authentic Leadership on Group Cohesion and Work Engagement in Student Organizations in Mexico (Doctoral dissertation, Regent University).

[5] Villarreal, J. (2019). Authentic Leadership: A Study of the Relationship Between Authentic Leadership and Moral Values (Doctoral dissertation, Regent University).

[6] Laschinger, H. K., Borgogni, L., Consiglio, C., and Read, E. (2015). The effects of authentic leadership, six areas of worklife, and occupational coping self-efficacy on new graduate nurses' burnout and mental health: A cross sectional study. International Journal of Nursing Studies; 52 (6): $1080-1089$. https://doi.org/10.1016/j.ijnurstu.2015.03.002.

[7] Baron, L. (2015). Authentic leadership and mindfulness development through action learning. Journal of Managerial Psychology; 31 (1): 296-311. http://dx.doi.org/10.1108/JMP04-2014-0135.

[8] Kiersch, C. E., \& Byrne, Z. S. (2015). Is being authentic being fair? Multilevel examination of authentic leadership, justice, and employee outcomes. Journal of Leadership \& Organizational Studies; 22 (3): 292-303.

[9] Xu, Z and Yang, F. (2019). The Cross-Level Effect of Authentic Leadership on Teacher Emotional Exhaustion: The Chain Mediating Role of Structural and Psychological Empowerment. Journal of Pacific Rim Psychology; 12 (35): 1-11.

[10] Kelly, C. D. (2019). The Relationship of Structural Empowerment, Job Satisfaction, and Intent to Leave in Critical Care Nurses (Doctoral dissertation, Grand Canyon University). 38-40.

[11] Almuhsen, F., Alkorashy, H., Baddar, F. and Qasim, A. (2017). Work environment characteristics as perceived by nurses in Saudi Arabia. International Journal of Advanced Nursing Studies; 6 (1): 45-55.

[12] Al Moosa, M., Hassanein, S. E., Alnems, A., Abdrbo, A. A., Minguez, O. and Ghadheeb, F. A. (2020). Perception of Nurses Work Environment in Tertiary Care Hospital in Saudi Arabia. American Journal of Nursing Science; 9 (1): 23-29.

[13] Ogata, Y., Sasaki, M., Yumoto, Y., Yonekura, Y., Nagano, M., and Kanda, K. (2018). Reliability and validity of the practice environment scale of the nursing work index for Japanese hospital nurses. Nursing open, 5 (3), 362-369.

[14] Davidson. E. S. (2017). Presence of Authentic Leadership and Bullying in the Nursing Workplace: A Correlational Study. Doctor's Degree; University of Arkansas for Medical Sciences.

[15] Alexander, C. \& Lopez, R. P. (2018). A thematic analysis of self-described authentic leadership behaviors among experienced nurse executives. The Journal of Nursing Administration, 48 (1), 38-43.

[16] Neider, L. L., \& Schriesheim, C. A. (2011). The authentic leadership inventory (ALI): Development and empirical tests. The Leadership Quarterly; 22: 1146-1164. doi: 10.1016/j.leaqua.2011.09.008.

[17] Laschinger, H. K. S., Shamian, J., and Thomson, D. (2001b). Impact of magnet hospital characteristics on nurse's perceptions of trust, burnout, quality of care and work satisfaction. Nursing Economics; 19 (5): 209 - 219. 
[18] Laschinger, H. K. S., Leiter M., Day, A. \& Gilin, D. (2009b) Workplace empowerment, incivility, and burnout: Impact on staff nurse recruitment and retention outcomes. Journal of Nursing Management 17, 302-311.

[19] Manojlovich, M., \& Laschinger, H. K. S. (2002). The relationship of empowerment and selected personality characteristics to nursing job satisfaction. Journal of Nursing Administration; 32, 586-595.

[20] Faulkner, J., \& Laschinger, H. K. S. (2008). The effects of structural and psychological empowerment on perceived respect in acute care nurses. Journal of Nursing Management; 16: $214-221$.

[21] Lake, E. T. (2002). Development of the practice environment scale of the Nursing Work Index. Research in Nursing and Health, 25, 176-188. https://doi.org/10.1002/nur.10032.

[22] Lee, H. F., Chiang, H. and Kuo, H. (2019). Relationship between Authentic Leadership and Nurses' Intent to Leave: The Mediating Role of Work Environment and Burnout. J Nurs Manag; 27: 52-65.

[23] American Association of Critical-Care Nurses (2005). AACN standards for establishing and sustaining healthy work environments: A journey to excellence. Retrieved from http://ajcc.aacnjournals.org/content/ 14/3/187.full.pdf + html.

[24] Azanza, G., Moriano, F., Molero, F., and Mangin, J. P. (2015). The effects of authentic leadership and organizational commitment on turnover intention. Leadership and Organization Development Journal; 37 (2): 181-199. https://doi.org/10.1108/lodj-05-2014-0090.

[25] Mason, J. C. (2019). Authentic leadership, employee engagement, and trust at a major logistics service company. Doctor of Education in Organizational Leadership; Pepperdine University.

[26] Haddad, L. M. (2013). "Generational Differences in Empowerment, Professional Practice Environment, Incivility, Authentic Leadership, Job Satisfaction, Engagement and Intent to Leave in Acute Care Nurses. " $\mathrm{PhD}$ diss., University of Tennessee, http://trace.tennessee.edu/utk_graddiss/1728.

[27] Regan, S., Laschinger, H. K. S. \& Wong, C. A. (2016). The influence of empowerment, authentic leadership, and professional practice environments on nurses' perceived Interprofessional collaboration. Journal of Nursing Management; 24: 54-61.

[28] Spence Laschinger, H. K., Wong, C. A. and. Grau, A. L. (2013). Authentic leadership, empowerment and burnout: a comparison in new graduates and experienced nurses. Journal of Nursing Management; 21: 541-552.

[29] Lucas V., Laschinger H. K. S. \& Wong C. A. (2008). The impact of emotional intelligent leadership on staff nurse empowerment the moderating effect of span of control. Journal of Nursing Management 16 (8), 964-973.

[30] Young-Ritchie, C., Laschinger, H. K. S. \& Wong, C. (2009). The effects of emotionally intelligent leadership behavior on emergency staff nurses' workplace empowerment and organizational commitment. Canadian Journal of Nursing Research; 22 (1): 70-85.
[31] Laschinger H. K., Wong C. A. and Grau A. (2012) Authentic leadership, empowerment and burnout: a comparison in new graduates and experienced nurses. Journal of Nursing Management. doi: 10.1111/j.1365-2834.2012.01375. x.

[32] Wong, C. A. \& Laschinger, H. K. S. (2013). Authentic leadership, performance, and job satisfaction: the mediating role of empowerment. Journal of Advanced Nursing; 69 (4): 947-959.

[33] Patrick A., Laschinger H. K. S., Wong C. \& Finegan J. (2011). Developing and testing a new measure of staff nurse clinical leadership: the clinical leadership survey. Journal of Nursing Management 19 (4), 449-460.

[34] Cheng, L., Cui, Y. Chen, Q., Ye, Y., Liu, Y. et al. (2020). Pediatric Nurses' General Self-efficacy, Perceived Organizational Support and Perceived Professional Benefits from Class A Tertiary Hospitals in Jilin Province of China: The Mediating Effect of Nursing Practice Environment. BMC Health Services Research; 20 (12): 1-9.

[35] Lin, C. F., Huang, C. I., Yang, C. M., \& Lu, M. S. (2019). The relationship between work environment satisfaction and retention intention among nursing administrators in Taiwan. The Journal of Nursing Research, 27 (5): 1-9.

[36] Ozer, O., Santas, F., Santas, G. and Sahin D. S. (2017). Impact of nurses' perceptions of work environment and communication satisfaction on their intention to quit. Int $\mathrm{J}$ Nurs Pract.; e12596. https://doi.org/10.1111/ijn.12596.

[37] Ulusoy, H., and Polatkan, R. (2016). Assessment of the nurses' work environment using the nursing work index scale. Cumhuriyet Medical Journal; 38 (4): 246-257. https://doi.org/10.7197/cmj.v38i3.5000196868.

[38] Mollaoglu, M., Fertelli, T. K., \& Tuncay, F. O. (2010). Assessment of perception related work environment of nurses working in hospital. Firat University Journals of Health Sciences; 5 (15): 17-30.

[39] Zhang, L. F., You, L. M., Liu, K., Zheng, J., Fang, J. B., Lu, M. M. and $\mathrm{Bu}, \mathrm{X}$. Q. (2014). The association of Chinese hospital work environment with nurse burnout, job satisfaction, and intention to leave. Nursing Outlook; 62 (2): 128-137. https://doi.org/10.1016/j.outlook.2013.10.010.

[40] Al Hadrawi, H., Al-Biaty, D., AL-Fayyadh, S., \& Alzeyadi, S. (2017). The link between nurse workplace environment and patient satisfaction with nursing care services. New Trends and Issues Proceedings on Humanities and Social Sciences. [Online]. 4 (2): pp 267-274. Available from: www.prosoc.eu.

[41] Washburn, K. D. (2017). Effects of Authentic Leadership Style and Nurse Engagement on Patient Satisfaction. Doctoral of Business Administration; Walden University.

[42] Khan, B. P., Griffin, M. T. Q., \& Fitzpatrick, J. J. (2018). Staff Nurses' Perceptions of Their Nurse Managers' Transformational Leadership Behaviors and Their Own Structural Empowerment. Journal of Nursing Administration; 48 (12): 609-614. 